\title{
A Study on the Consultation Work of National Physical Fitness Measurement
}

\author{
Xiaodan Chi \\ Physical Education College, Leshan Normal University, Leshan, 614000, China
}

Keywords: National physical fitness. Measurement. Consultation work

\begin{abstract}
Physical fitness measurement and assessment are all important in the work of national physical fitness measurement, in which measurement is the basis while assessment is the key point. Researches on the feelings and experiences of the people who have participated in national physical fitness measurement in recent years have been done to continuously improve the quality of the physical fitness measurement. In parallel with the consultation work, the staff must have the professional quality and know the principles of consultation. In this thesis, the principles that need to be applied during the consultation process, on-site consultation requirements and so on are discussed, and then some reasonable suggestions for the consultation work of national physical fitness measurement are put forward.
\end{abstract}

\section{Introduction}

National physical fitness measurement functions as a way to communicate and consult with people face-to-face, understand the physical condition, physical function, quality and other aspects of the individual citizens, and provide reasonable suggestions for healthy exercise, which aims to raise people's enthusiasm for physical exercise. Professionals can learn about people's physical condition by consultation, and can give reasonable guidance by consulting their values of living and living habits, their knowledge about nutrition, exercises, and etc. So such consultation work is not superficial but gives play to the vital function of the measurement work, so as to strengthen people's physical fitness. The definition and significance of national physical fitness measurement are analyzed in the following.

\section{Definition of Consultation Work of National Physical Fitness Measurement}

\section{Definition of Consultation}

Consultation is a method of offering people certain help through mutual exchanges, which mainly includes verbal and nonverbal ways. During the process of national physical fitness measurement, professionals can analyze the consultation results and then help the participants to solve corresponding problems, such as matters that deserve attention in diet and fitness exercises. Also, consultation results, when materialized in the form of report, will be analyzed from the viewpoint of the consulter, for the purpose of improving participants' knowledge. Participants can be individuals or families, with certain variability.

\section{Main Methods of Consultation Work}

Main ways of consultation work include the following points:

Firstly, face-to-face communication with professionals. It is a relative common way for consultation. Professionals mainly communicate with consulters on the spot, and analyze their condition in time, which has certain timeliness.

Secondly, telephone counseling. Consulters put forward corresponding questions to professionals through telephone, and professionals get to know the actual situation of consulters in the conversation and give reasonable suggestions and guidance.

Thirdly, column counseling. Professionals publish relevant notice to inform consulters to question through newspaper, radio station, TV station and internet columns, and professionals answer the questions within a certain period of time. 


\section{Main Theory of Consultation Work}

The main theory of consultation work is to change consulters' living habits through consultation, and to help them form healthy and green values of living and good living habits in daily life by guiding consulters with different living habits. For example, when consulters consult about healthy exercise, professionals should inform them of information about exercise attitude, values, sport skills, exercise prescription and etc, so as to change the way they used to exercise.

\section{Importance and Significance of Consultation}

The consultation work of national physical fitness measurement can not only help consulters understand their own physical condition, but also constantly change their values of living and living habits and make them get deeper understanding of nutrition and healthy exercise, which give an effective play to the role of national physical fitness measurement and so as to promote people's physical health. The importance of consultation lies in that the quality of consultation work has great influence on the understanding of national healthy physical fitness, and represents the strength of National Physical Fitness Surveillance Center.

The content of national physical fitness measurement consists of interpretation of the testing results and suggestions by professionals. Professionals should make different suggestions according to the actual situation of consulters. The significance of consultation work of national physical fitness measurement includes the following points:

Firstly, it helps the consulters know the indicators of the test

Secondly, it makes the consulters understand their own condition in accordance with the content of the indicators.

Thirdly, Professionals discuss with the consulters and analyze the testing results and its reason.

Lastly, it provides the consulters with reasonable advices, such as matters needs attention in nutrition, exercise and exercise skills and etc. Moreover, it helps them form healthy and green values of living by mutual exchanges.

\section{The principles that Need Attention during the Consultation Process}

The main principles of consultation lie in that consulting staff should abide by the requirements of consultation work, constantly practice in the consultation work and accumulate relevant experiences during their work. The principles that need attention during the process of consultation are analyzed in the following.

\section{The Principle of Voluntariness}

National physical fitness measurement is for the sake of assessing individuals' physical condition, and guiding them to exercise through reasonable methods, thus rectifying their unhealthy living habits. The consultants must work in accordance with the will of the consulters and should not put forward pressure on them. Professionals and the consulters are equal in the course of their work, so they should give friendly response rather than discriminatory one. Moreover, the consulters put forward questions in a spontaneous way. Only when these requirements are met, the consultation results can be effectively improved.

\section{Principle of Making Friends through Conversation}

Consultants should establish a trusting relationship with the consulters in the conversation, through which the consulters can believe consultants so as to contribute to the smooth conduct of the consultation. In the consultation process, the most effective method is to communicate like friends. By understanding their physical condition and concerning about their daily habits from a friend's point of view, the consultants can make reasonable health plans with an exploring attitude.

\section{Principle of Flexibility}

Some consulters in the consultation process will deliberately avoid bad conditions, or some consultants question in a too straightforward way, leading to the discontent of the consulters, which is connected to the flexibility of inquiry skills of the consultants. To achieve good results, it is necessary to pay attention to expression skills, to use flexible way of inquiry and communication steps, to 
change the way of communication according to the actual situation of different consulters so as to avoid communication barriers caused by inflexibility in the consultation process.

\section{Principle of Confidentiality}

In the process of consultation, the principle of confidential must be noted. The consulters will inevitably reveal their privacy in the test. If the privacy is disclosed, the consulters' right of privacy will be hurt. It is necessary for the consultants to protect and respect the privacy of the consulters morally and legally, for it is a reflection of professional ethics and responsibility in the work.

In the consultation process, people will reveal their privacy out of respect for the consultants, but the consultants' discussing and joking at the privacy will seriously affect the image of the consulters, which would cause public discontent and even legal disputes. Therefore, the consultants should take good care of the relevant materials, avoid discussing the consulters in their spare time, and not disclose the consulters' names, home addresses, etc. in any occasion.

Foremost, the names of the consulters can not be published in newspapers and magazines, which should be replaced by pseudonyms. Also, the expression skills should be laid stress on to avoid privacy disclosure.

Besides, the consulters' privacy can not be discussed in any occasion. But the privacy discussion can be heard as a result of the consultants' low moral quality. So In order to ensure the interests of the consulters, we must establish regulations prohibiting staff to communicate privacy issues with colleagues, friends and relatives.

At last, in addition to the personnel of the department, the relevant departments who want to get access to consulters' privacy should go through legal approach, like showing certificate provided by public security organs.

\section{Principle of Individuality}

Due to different living and work environment, people's hobbies and habits differentiate. In the consultation process, the most important work of the consultants is to understand the mass work and help people develop reasonable healthy exercise programs to meet individual needs.

In the process of consultation, the consultants should put forward pertinent advices with appropriate tone and attitude and instill ideas of healthy sports for the consulters, thus helping them live and work in a proper way. By telling the truth and facts to the consulters, they let the people face their own problems more positively, and form positive and optimistic spirit.

\section{Analysis of the Common Methods in Consultation Work}

This thesis analyzes the common methods, common equipments, common indicators in consultation according to the content of consultation, and puts forward a few reasonable suggestions.

\section{Physical Fitness Test}

Physical fitness test is one of the basic methods of national physical fitness measurement, which is designed mainly according to the national body structure, physical fitness and so on. It includes the following key indicators:

Foremost, it analyzes the indicators of people's body shape and structure, and the proportion of their height and weight, body type, nutrition and so on.

Moreover, it analyzes people's sport quality consisting of grip strength, back strength, sit-ups, left-right cross, explosive power, muscular strength and endurance and so on. Also, the coordination, flexibility, cardiovascular endurance of people are analyzed to determine the basic condition of sport quality and rehabilitation level.

The instruments used include: instruments of national physical fitness measurement, Helmas Health Management System, CSTF Physical Fitness Tester. These testers can also be a reminder for the participants, and calculate their sport target heart rate, physical strength, health age and so on.

Lastly, it analyzes the indicators of physiological functions which include pulse, blood pressure, lung capacity, oxygen uptake and so on. The test of participants' physiological functions can reflect the efficiency of their metabolism and cardiovascular system.

\section{Rating of the Testing Results}


The testing results of consulters can be detected in accordance with health standards, evaluated according to standards of physical fitness measurement, and analyzed and interpreted in accordance with the relevant measurement by working staff. The detailed descriptions are in the following.

Firstly, interpret the Sport Target Heart Rate. Sport target heart rate is the heart rate when people reach the best fitness effects during exercise. Heart rate has a close relationship with body condition and age. In daily exercise, keeping exercise for 15 minutes or so, which is the exercise intensity of Sport Target Heart Rate, we can achieve the best fitness effects, being beneficial to health.

Secondly, rate the comprehensive physical fitness of people. According to the grade, we can understand the physical condition of the consulters. The main analysis is as follows: Excellent: $\geqslant 80$ points, it is on behalf of groups of regular physical exercise, with good physical condition and vigor; well :76-70 points, it refers to groups that have a more healthier lifestyle and regular exercises, with good physical condition; General :71-75 points, it stands for the groups that have irregular exercises, declined health indicators, and are in the sub-health state; poor: $\leqslant 70$ points, it represents the groups that are in the sub-health state, lack of care, and have sharply declining health indicators.

Thirdly, rate the health age for people. Health age refers to the age and physical conditions reflected by related performance. Health age is not consistent with the actual age, which is mainly a generalization of the individual's health conditions. When the health age is less than the actual age, the individual has strong adaptability and healthy physique with vigor; on the contrary, it is a representative of premature aging.

\section{Key Points of Consultation Work}

After the rating, the staff must analyze the consultation work as well as individual's nutrition, law of life, disease and other aspects of physical fitness. Inadequate physical fitness represents the individual's declined mobility, lack of adaptability for the changing environment in terms of metabolism and resistance to the natural world, which leads to the sub-health state. Thus, in the course of consultation, the staff should adopt different solutions:

Firstly, As to consulters have better physical fitness, the staff should affirm their results of exercise, encourage them to more participate in healthy exercises, use scientific means and methods to help them develop a reasonable exercise program to strengthen their physical fitness.

Secondly, for consulters with general physical fitness, the staff should strengthen the advocacy of exercise to make them exercise more actively, and regularly communicate with the participants, encouraging them to exercise several times a week in order to enhance their physical fitness.

Thirdly, regarding to consulters with poor physical fitness, the staff should point out the main reason for the serious problems in their lifestyle, encourage them make timely adjustments in their daily life and make exercise several times a week to improve the physical fitness.

\section{Conclusion}

In the course of the test, the staff often encounter a variety of patients, whose bad physical conditions are largely caused by lack of physical exercise. So during the consultation process, the staff should pay attention to this group of consulters, make explanation for them through their own professional knowledge to help improve consulters' alertness, strengthen physical exercise, and distribute reasonable diet. Meanwhile, during the course of conversation, the staff should enhance the participants' confidence, take care of their health, and communicate with them. Consequently, they can really trust the staff, and cooperate to take exercise.

\section{References}

[1] Zhang Yihong: A Study on the Characteristics of Quasi Public Goods and Its Supply of National Physical Fitness Measurement Service, Physical Education and Science, 2008.06 (02).

[2] Zhu Xiaoping: Chatting Medical Science---obesity, Family Medical Science, 2009.02 (04).

[3] Zhang Yihong.: The Application of Measurement of PWV and ABI in National Physical Fitness Measurement, Journal of Physical Education, 2009.03 (7). 